\title{
Digital Holographic Three-Dimensional Video Displays
}

Included in this paper are a signal-processing-based analysis of light diffraction by spatial modulators, pickup schemes for these displays, and specifications for a satisfactory quality display.

\author{
By Levent Onural, Fellow IEeE, Fahri Yaraș, Member IEEe, and Hoonjong Kang
}

\begin{abstract}
Holography aims to record and regenerate volume filling light fields to reproduce ghost-like 3-D images that are optically indistinguishable from their physical 3-D originals. Digital holographic video displays are pixelated devices on which digital holograms can be written at video rates. Spatial light modulators (SLMs) are used for such purposes in practice; even though it is desirable to have SLMs that can modulate both the phase and amplitude of the incident light at each pixel, usually amplitude-only or phase-only SLMs are available. Many laboratories have reported working prototypes using different designs. Size and resolution of the SLMs are quite demanding for satisfactory 3-D reconstructions. Space-bandwidth product (SBP) seems like a good metric for quality analysis. Even though moderate SBP is satisfactory for a stationary observer with no lateral or rotational motion, the required SBP quickly increases when such motion is allowed. Multi-SLM designs, especially over curved surfaces, relieve high bandwidth requirements, and therefore, are strong candidates for futuristic holographic video displays. Holograms are quite robust to noise and quantization. It is demonstrated that either laser or light-emitting diode (LED) illumination is feasible. Current research momentum is increasing with many exciting and encouraging results.
\end{abstract}

KEYWORDS I Digital holography; holographic video; spatial light modulators (SLMs); 3-D displays; 3DTV

\footnotetext{
Manuscript received March 26, 2010; revised September 9, 2010; accepted November 26, 2010. Date of publication February 4, 2011; date of current version March 18, 2011. This work was supported by the European Commission (EC) within FP7 under Grant 216105 with the acronym Real 3D.

L. Onural and F. Yaraș are with the Department of Electrical and Electronics Engineering, Bilkent University, TR-06800 Ankara, Turkey (e-mail: onural@bilkent.edu.tr; fahri@ee.bilkent.edu.tr).

H. Kang was with the Department of Electrical and Electronics Engineering, Bilkent University, TR-06800 Ankara, Turkey. He is now with the Korea Electronics Technology Institute (KETI), Gyeonggi 463-816, Korea (e-mail: hjkang@ee.bilkent.edu.tr).

Digital Object Identifier: 10.1109/JPROC.2010.2098430
}

\section{INTRODUCTION}

Three-dimensional video displays that can generate ghostlike optical duplicates of 3-D objects and scenes have been depicted in science-fiction movies as futuristic means of visual media tools; such display devices always attracted public interest [1].

One immediate question is whether such a display is possible; and a quick answer is "Yes, it is." Noting that "seeing" is a purely optical interaction, and what we (or any other observer, including living organisms and machines) see is only due to the light that enters through our pupils, the design target for such a display is simple to state: if we can record the volume filling time-varying light field in a 3-D scene, with all its needed physical properties, and then regenerate the same light field somehow at another place, maybe at another time, the observer will not be able to distinguish the original scene from its duplicate since the received light will be the same, and therefore, any visual perception will also be the same.

Then the natural question is whether we can record the light with all its relevant physical properties, and then regenerate it. The classical video camera is also a light recorder. However, not all necessary physical properties of light for the purpose outlined above can be recorded by a video camera; indeed, what is recorded by a video camera is just the focused intensity patterns (one for each basic color) over a planar sensing device. What is needed to be recorded instead is indeed much more complicated: we also need the directional decomposition of incoming light as well. Briefly, and in an idealized sense, we can say that we need to record the light field distribution. The term light field distribution is usually associated with ray optics concepts, and therefore, can be a valid optics model only in limited cases. If it can be recorded, we then need physical 
devices that can also regenerate (replay) the recorded light field. Prototypes for light field recording and rendering devices are reported in the literature [2]. Integral imaging gets close to a light field imaging device in the limit under some mathematical idealizations; however such limiting cases are not physically possible [3]. A better optical model than the ray optics is the wave optics. The propagation of light in a volume is modeled as a scalar wave field; the optical information due to a 3-D scene is carried by this wave field. Therefore, if such a wave field can be recorded and replayed, we achieve visual duplication of 3-D scenes; this is holography [4]. Scalar wave model is usually satisfactory, and more accurate models of light are rarely needed, if any, for 3-D imaging and display purposes.

Therefore, the term holography refers to recording and replaying optical wave fields. In a more restrictive usage, holography refers only to a specific form of such recording where interference of the desired wave field with a reference wave (sometimes self-referencing is employed, as in in-line holography) is formed and recorded; we prefer the broader usage as stated above. Indeed, the usage of the term may even be further broadened to include all kinds of physical duplication of light, and therefore, may also cover integral imaging, in a sense [1].

Here in this paper, our focus is on the display of holograms. We focus only on dynamic displays for video. Still holographic display technology has been well developed since 1960s, whereas dynamic display technology is still in its infancy, and therefore, a current research topic. We further restrict our focus to pixelated display devices that can be driven digitally. Such displays are usually called digital electroholographic displays since they are driven electronically.

An overview of some research results in this field, together with current research interests and achievements, will be presented in Section II. Section III presents an analysis to understand the effects of different parameters to the holographic reconstruction quality; the analysis then leads the specifications of a satisfactory quality digital dynamic holographic display. Conclusions are drawn in Section IV.

\section{STATE OF THE ART IN DYNAMIC HOLOGRAPHIC DISPLAYS}

\section{A. Overview}

Even though we focus on dynamic holographic displays in this paper, we feel that it is appropriate to start with a brief history of holography in general.

Gabor (1900-1979) invented the holography to reduce the aberrations in electron microscopy [5]-[7]. However, due to low quality of obtained images holography did not become popular until early 1960s. After the developments in laser technologies, Leith and Upatnieks [8]-[10] developed the off-axis holography. In the meantime, Denisyuk invented the volume holography by bringing the work of Lippmann to holography [11], [12]. Still holography has been significantly developed since then, and many excellent monochromatic and color holograms have been made.

The first computer generated hologram was introduced by Lohmann and Paris in 1967 [13]. In the same year, Goodman and Lawrence brought forward the idea of the digital holography [14]. Then, in 1980, the fundamental theory of digital holography was introduced by Yaroslavskii and Merzlyakov [15]. We use the term "digital holography" in a broader sense to include all sorts of digital techniques to compute wave propagation, diffraction, and interference, as well as, digital capture and digital display of holograms.

Conventional thick holograms on photographic plates can provide high resolution and full parallax. However, dynamic displays for holographic video are still far from providing satisfactory results. In electroholography, the resolution is significantly lower compared to thick holograms. Moreover, pixelated structures bring some additional problems. Pixel period determines the maximum frequency that can be represented when digital-to-analog conversion is conducted in the Shannon sense, and this in turn determines the maximum diffraction angle as outlined in Section III-A2. Unfortunately, the pixel periods are not currently small enough to support sufficiently large viewing angles. Problems associated with pixelated electroholographic display are known [16].

Since liquid crystal spatial light modulators (SLMs) are currently the primary choice for digital holographic displays, it is quite relevant to briefly mention current capabilities of such devices. Bauchert et al. [17] reported the desirable features of liquid crystal SLMs. These features can be summarized as higher number of pixels, smaller pixel period, better optical efficiency, and faster operation. There are various SLMs such as liquid-crystal-based devices (liquid crystal devices and liquid crystal on silicon devices), mirror based devices (digital micromirror devices) and solid crystal devices (acousto-optical devices). The acousto-optical modulators (AOMs) are mostly used in 1-D applications. The digital micromirror devices are usually for binary modulation and they may result in additional noise due to vibration of micromirrors. The liquidcrystal-based light modulators are more commonly used in electroholographic applications. Michalkiewicz et al. presented the progress in liquid crystal on silicon (LCoS) SLMs and their applications [18]. Ohmura et al. proposed a method to increase the viewing angle using such SLMs [19]. In their proposed system, they used a single SLM that was driven by a mirror module. As a consequence of this method the resolution along the horizontal direction increases. Therefore, the horizontal diffraction angle also increases; and thus the viewing angle is improved.

Liquid-crystal-based SLMs are classified into various types such as complex amplitude, amplitude-only, phaseonly, transmissive- and reflective-type SLMs, and so on. 
The discussions corresponding to the bandwidth restriction and the pixel period given in Section III are valid for all such types of pixelated SLMs. Among them, the fully complex amplitude-type SLM may be the ultimate solution for the accurate reproduction from the hologram corresponding to a 3-D object. Ability to support complex functions at the display is highly desirable since diffraction fields are represented as complex valued fields where both the amplitude and the phase are needed. An ideal SLM pixel should modulate both the amplitude and the phase of the incident light. However, it is difficult to manufacture the complex amplitude-type SLMs based on current technology. Phase-only SLMs may be the next best solutions for electroholography because they have several advantages over amplitude-only SLMs such as suppressed zeroth-order and high-diffraction efficiency, which can theoretically reach $100 \%$. Amplitude-only SLMs can also be used for electroholography. However, problems associated with strong undesired diffraction orders are more severe compared to the phase-only case. A research group from Barcelona University, Barcelona, Spain, combined two SLMs to display full complex Fresnel holograms [20]-[22]. They used one SLM for the amplitude and the other one for the phase. They also investigated the quality of the reconstructions using real-only, imaginary-only, amplitude-only, and phase-only holograms.

Schwerdtner et al. reported a novel hologram technology, which they called tracked viewing window (TVW) [23]-[27]. By this approach they only calculate a small portion of a hologram, which then reconstructs a narrow angle light that falls onto the tracked pupils of the observer. They demonstrated that thin film transistor (TFT) monitors can then be used as SLMs to build holographic displays.

Another electroholographic display technique was presented by Hahn et al. [28]. In their research, they used curved array of SLMs to increase the field of view.

Spatial Imaging Group at the Massachusetts Institute of Technology (MIT, Cambridge, MA) developed a series of holographic display systems named Mark-I, Mark-II, and Mark-III [29]-[36]. Mark-I and Mark-II use acoustooptical modulators, whereas Mark-III uses guided-wave optical scanners. All three can render 3-D objects at video rates. A company developed another holographic display system [37]. The system uses active tiling where an electrically addressed SLM (EASLM) projects tiles of a big hologram onto an optically addressed SLM (OASLM). With the help of the setup, more than 100 megapixels holograms can be displayed. Another system, so-called Horn (HOlographic ReconstructioN), was presented by a group in Chiba University, Chiba, Japan [38]-[44]. Fieldprogrammable gate arrays (FPGAs) were used in the developed holographic display system to achieve video frame rates. Another group from Japan also demonstrated a holographic display system [45]; the system at the National Institute of Information and Communications Technolo- gies (NICT, Tokyo, Japan) captures the 3-D scene by an integral imaging camera. The digital holograms of the captured scene is calculated and displayed in real time.

For further details, the reader is referred to a broad survey on dynamic holographic displays, which was recently published [46].

\section{B. Recent Results From Bilkent University}

Holographic displays have been investigated at Bilkent University, Ankara, Turkey, since early 1990s [47]. Recently, they used SLMs for such purposes and demonstrated single and multiple SLM holographic displays. They mostly use phase-only SLMs. For example, in a study involving only one phase-only SLM [16], in-line phase holograms, which were calculated by Gerchberg-Saxton algorithm [48], were used to show that reconstructions that are larger than the SLM size are feasible. In another system, three SLMs were used to generate color holographic reconstructions [49]. Again the Gerchberg-Saxton algorithm was used to generate the in-line phase holograms that were written on the SLMs. Three phase holograms were calculated separately (for red, green, and blue channel) and loaded to the SLMs. Color light-emitting diodes (LEDs) were used as light sources; all three reconstructions were combined to obtain a color reconstruction. Yet another system generates and displays holograms in real time [50]-[52]. The phase-only holograms for the display were computed using a fast, approximation-based algorithm called accurate compensated phase-added stereogram (ACPAS) [53]-[56], which was implemented on graphics processing units (GPUs) to render the holograms at video rates. LEDs were used as light sources for reconstructions that can be observed by naked eye. Fig. 1 shows the overall setup for the real-time color holographic display system and Fig. 2(a)-(c) shows the original color 3-D model, the computer reconstruction from the phase-only hologram, and the optical reconstruction from the same hologram written on the SLM, respectively. They also compared the quality of optical reconstructions obtained by using a laser and a LED as the light source [50]. Even though LEDs have broader spectra than lasers, they conclude that reconstructions using LEDs can be still satisfactory in quality. In a recent prototype, a curved array of six phase-only SLMs was used to increase the field of view. As a consequence of the achieved large field of view, the observer can look at the optical reconstruction binocularly and see a real 3-D image floating in the space. Reconstructions can also be observed from different angles without any discontinuity and with a larger horizontal parallax. Fig. 3 shows the optical reconstructions of a pyramid recorded from different angles. The ghost-like 3-D image (a real optical image) was positioned next to a similar physical object located at the same depth, and the recording camera was focused to that plane; such a setup shows the depth location of the reconstruction, as well as its quality of parallax and sharpness by providing a similar 


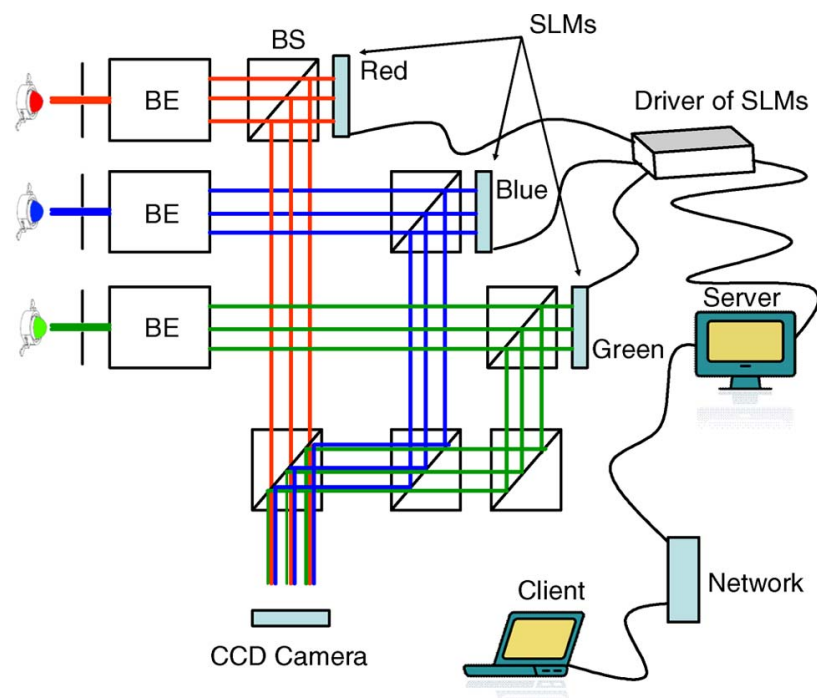

Fig. 1. Overall setup: BE_ beam expander; BS — nonpolarized beam splitter.

physical object for comparison. The actual size of the base of the pyramid is about $1 \mathrm{~cm} \times 1 \mathrm{~cm}$, and its height is about $2 \mathrm{~cm}$. The reconstruction (real image) is about $50 \mathrm{~cm}$ in front of the SLMs.

This brief overview of current state of the art indicates that dynamic holographic displays do have the potential for highly satisfactory futuristic 3DTV displays; however, they do not yet provide such satisfactory results to the consumer who expects the counterpart of crisp clear conventional 2DTV displays. Further research is certainly needed.

A detailed survey of the state of the art in dynamic holographic displays was recently published by Yaraş et al. [46].

\section{SPECIFICATIONS OF A}

\section{SATISFACTORY QUALITY}

\section{DYNAMIC HOLOGRAPHIC DISPLAY}

The design and implementation of electronically controllable dynamic displays to support holographic video are the

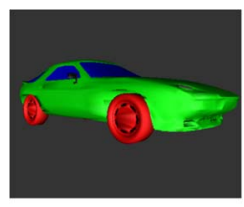

(a)

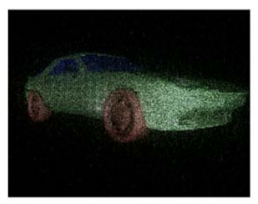

(b)

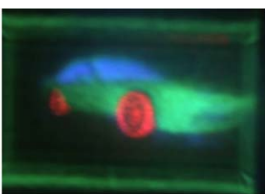

(c)
Fig. 2. Color holographic reconstruction using SLMs. (a) A rigid color 3-D object. (b) Computer reconstruction from the hologram of the 3-D object. (The hologram was calculated by using the ACPAS algorithm.) (c) Optical reconstruction (a single frame of the holographic video) [50].

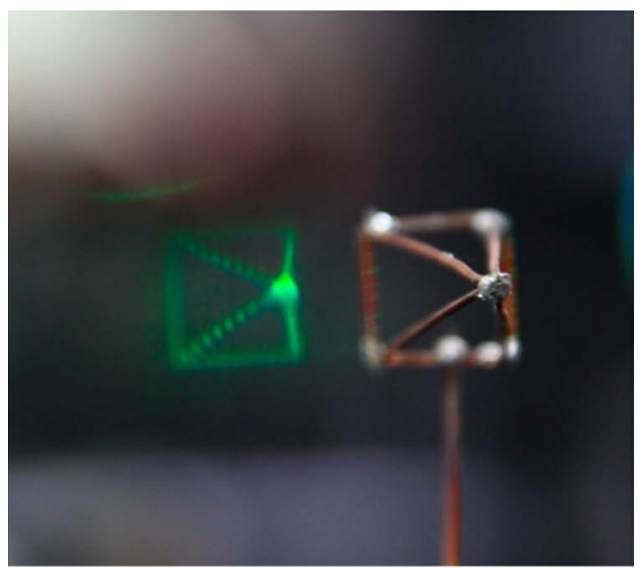

(a)

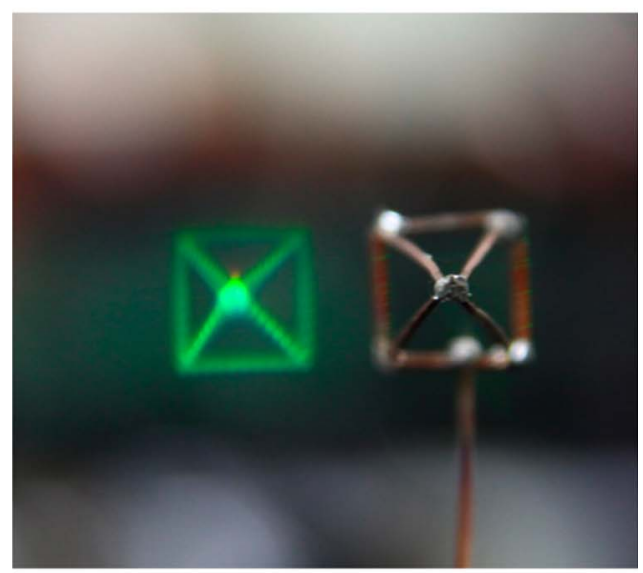

(b)

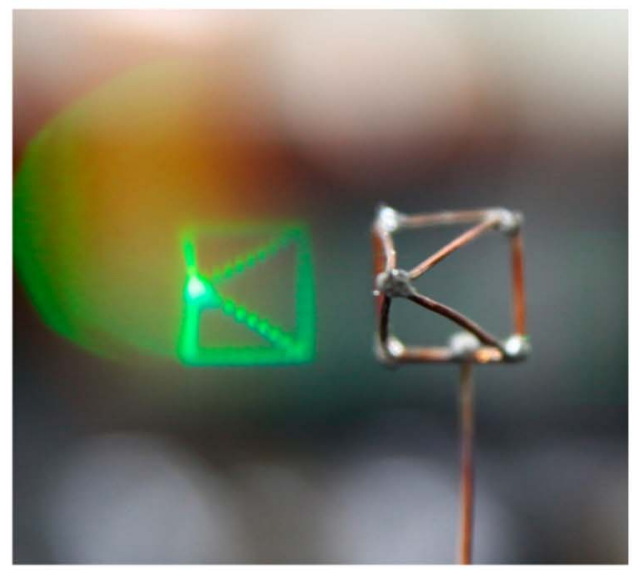

(c)

Fig. 3. Optical reconstructions from a curved array of SLMS: (a) left, (b) front, and (c) right view.

key issues for the success of such true 3-D displays. Currently available devices have quite limited capabilities, and thus, do not yield satisfactory performance, yet. We expect that such products will be significantly improved in the future. One of the tasks related to digital holographic video displays is to find the specifications associated with 
various physical parameters of a digital holographic display system to achieve satisfactory quality 3-D reconstructions. Candidate devices are different variants of pixelated SLMs for digital operation. Here in this section we present such specifications, for a few different cases, based on associated analysis and experiments. The analysis is based on related human visual system properties, which are then reflected to the parameters of satisfactory 3-D reconstructions; subsequent association of these reconstructed image features to the SLM parameters completes the analysis.

It is instructive to state some relevant specifications of classical analog photographic films that yield satisfactory still holographic displays. In order to create a faithful reproduction of the 3-D image of a physical object, the holographic recording material is required to have a high sensitivity and a high resolution. The spatial resolution of the finest hologram material is reported as many thousands of lines per millimeter; the diffraction efficiency of this material goes up to $98 \%$. Still hologram sizes of about $10 \mathrm{~cm}$ are common; sizes may go up to many tens of centimeters or even higher. Such size and resolution properties are sufficient to reproduce high-quality 3-D images of physical objects in natural color and texture.

\section{A. Size and Bandwidth Specifications for a Satisfactory Electroholographic Display}

We will base our discussion on the space-bandwidth product (SBP) that should be supported by a holographic display device and, in turn, by propagating optical waves that emanate from such a device. Furthermore, we will take the capabilities of the human visual system as our starting point in the analysis. The physical parameters of the human visual system, such as the field of view, spatial resolution, visible spectrum, and so on, are naturally limited. Therefore, the spatial bandwidth of an electroholographic display device may also be limited based on the corresponding limitations of the human visual system. A simple schematic for an electroholographic display system is shown in Fig. 4. A plane wave illuminates the hologram. This beam is diffracted by the SLM, with an area of $S_{H}$, towards multiple directions within an angle $2 \theta_{\max }$.

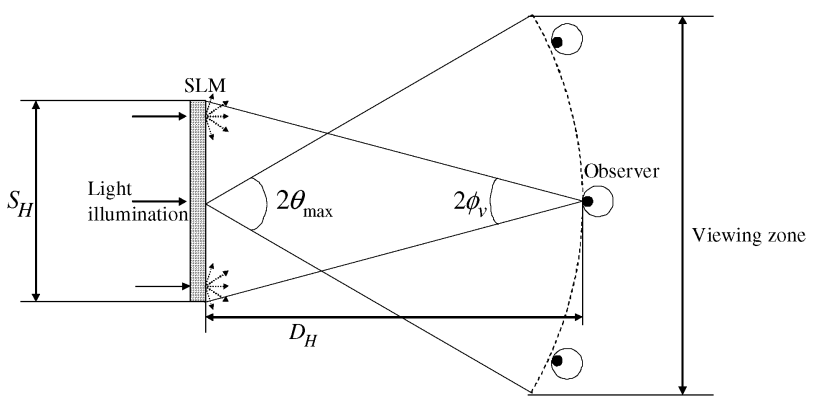

Fig. 4. Simple schematic for an electroholographic display.

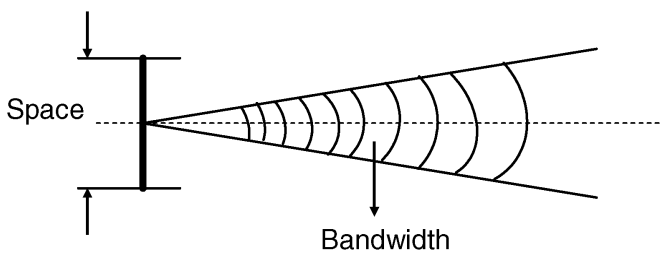

Fig. 5. The "space" and "bandwidth" to compute the SBP.

The distance between the observer and the hologram is denoted as $D_{\mathrm{H}}$ and the angular field of view of the observer is $2 \phi_{v}$. This schematic will be used to find the related specifications.

1) SBP of Electroholographic Displays: We first discuss the SBP, which seems like a good metric to assess the quality of holographic displays. Though it is an idealization, we assume that a hologram plane can be associated with a local frequency bandwidth at its each point as shown in Fig. 5. We further assume that the SLM should have a constant local frequency band throughout its surface. It is natural to expect that a higher SBP should lead to a higher quality in the reconstruction. Even though the figures and the related analysis are in 1-D for convenience, final results are for 2-D hologram shapes where the bandwidth is the area of the 2-D band over the frequency plane associated with 2-D holographic patterns. The consequences of bandwidth and the space limitations of an electroholographic display should be understood. Bandwidth restriction on a hologram is equivalent to propagation angle restriction of the plane waves propagating away from the hologram since each propagating wave will correspond to a 2-D complex sinusoidal pattern on the hologram plane. Therefore, this propagation angle constraint restricts the "sharpness" of the reconstructed object since higher propagation angles carry high-frequency contents of the reconstructed image. It is easy to assess the quality degradation due to propagation angle restriction: the angle restriction is equivalent to low-pass filtering. If we assume that our SLMs have small enough pixel period to support high propagation angles, the bandwidth restrictions should then come from the human visual system. The studies about human visual system show that the field of view $2 \phi_{v}$, in which the retina has the maximum resolution, is between $2^{\circ}$ and $5^{\circ}$ [57][59]. Being aware of different approaches to the subject in the literature, we set the value of such high-resolution field of view as $3^{\circ}$ for our analysis. This does not mean however that the vision outside the maximum resolution field of view, as described above, does not exist. Simply, the resolution of the perceived image, derived from the intensity on the retina, degrades for higher viewing angles, but a low-resolution image may still extent to $100^{\circ}-140^{\circ}$ of viewing angle and called peripheral vision [57], [59]. Therefore, for a realistic holographic reconstruction that 
would give the sense of being immersed in a natural environment, even for a stationary observer, quite high angles are still needed to be supported by the hologram. This in turn translates to very small pixel periods. However, we still start our analysis for a stationary observer whose field of view is restricted to the highresolution view with a viewing angle of $2 \phi_{v}=3^{\circ}$. Such an analysis will result in requirements of a holographic display, which would yield a high-resolution image under tunnel vision; the result is known as the keyhole effect. This restriction will then be removed as we proceed to support a moving and rotating observer. Furthermore, we assume that the resolution on the retina is circularly symmetric.

Therefore, we start with a stationary observer whose viewing angle is restricted to be $3^{\circ}$ and circularly symmetric. Such a restriction is equivalent to a lowbandwidth signal restriction on a 2-D cross section of the reconstructed image over the transversal plane. Moreover, the same low-bandwidth restriction is valid for any transversal plane, including the hologram plane, since wave propagation between parallel planes is a 2-D linear shift invariant operation [60]. In other words, diffraction angles higher than the field of view are not needed to be supported by a planar hologram for a stationary observer (no lateral or rotational motion of the observer). Therefore, maximum local diffraction angle of the light emanating from the hologram during the reconstruction should be chosen as equal to field of view $\left(\theta_{\max }=\phi_{v}\right)$. Having that maximum local diffraction angle of the hologram, we can calculate the maximum spatial frequency of the hologram as

$$
f_{\max }=\sin \left(\theta_{\max }\right) / \lambda
$$

which is the matching bandwidth limitation to the propagation angle restriction. For a stationary observer, the needed hologram size can be calculated as a function of distance between the eye and the hologram. Fig. 6 shows

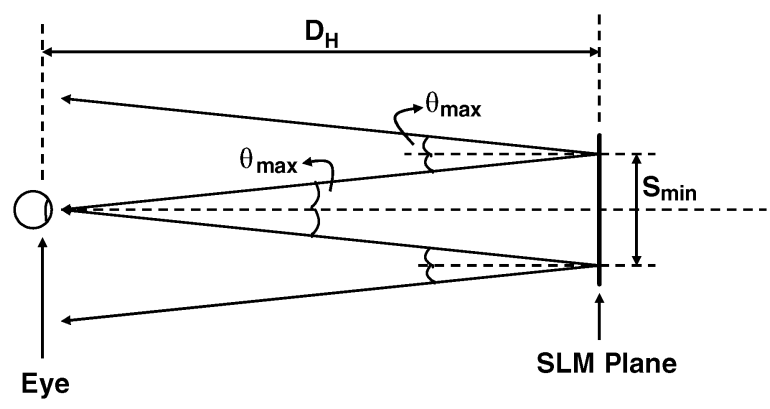

Fig. 6. Hologram size for a stationary observer. ( $D_{H}$ : Distance between hologram and eye. $S_{\min }$ : Hologram extent.)

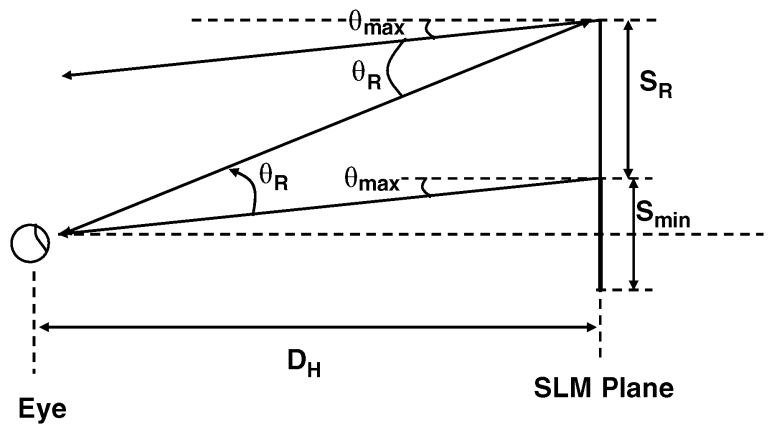

Fig. 7. Hologram size and bandwidth modification for eye rotation. $\left(D_{H}\right.$ : Distance between hologram and eye. $S_{\min }$ : Hologram extent for a stationary observer. $S_{R}$ : Additional hologram extent as a result of eye rotation. $\theta_{R}$ : Rotation angle in lateral direction.)

the relation between the maximum local diffraction angle and the hologram size. Then, the extent of the hologram in one dimension is

$$
S_{\min }=2 \tan \left(\theta_{\max }\right) D_{H}
$$

Thus, we get the needed SBP of a 1-D SLM to support a stationary observer as $2 f_{\max } S_{\min }$, where, $f_{\max }$ and $S_{\min }$ are as given by (1) and (2). Therefore, a holographic display with the specified size and bandwidth will support the highest resolution holographically reconstructed 3-D image that would fit to the highest resolution viewing range of a stationary observer.

However, if the observer is free to move along the lateral direction by $S_{O}$, hologram size should be modified accordingly. Then, the resultant needed hologram size to support a human observer who can laterally move (without any rotation) a distance of $S_{O}$ will be

$$
S_{H}=S_{\min }+S_{O}
$$

If we want the display system to also support a rotational motion of the human observer by $\theta_{R}$, another modification is needed as well. As seen in Fig. 7, both the hologram size and the bandwidth have to be modified to support rotation. The new hologram size should then be $S_{H}=S_{\min }+2 S_{R}$ where $S_{R}=\tan \left(\theta_{R}+\theta_{\max }\right) D_{H}-S_{\min } / 2$ and new bandwidth, for the given rotation angle is $f_{\max }=\sin \left(\theta_{R}+\right.$ $\left.\theta_{\max }\right) / \lambda$. As a conclusion, for an observer that is free to move along the lateral direction by $S_{O}$ and free to rotate up to an angle of $\pm \theta_{R}$, the resultant hologram extent should be $S_{H}=S_{\min }+S_{O}+2 S_{R}$. Moreover, the maximum frequency will be $f_{\max }=\sin \left(\theta_{R}+\theta_{\max }\right) / \lambda$, and the related bandwidth will be $2 f_{\max }$. Therefore, it means that if we insist on a planar holographic display, we will need 
significantly more bandwidth to support rotation. Again, we get the needed SBP to support such a translational and rotational motion of the observer as $\left\{2\left(S_{\min }+S_{O}+\right.\right.$ $\left.\left.2 S_{R}\right)\left[\sin \left(\theta_{R}+\theta_{\max }\right) / \lambda\right]\right\}$ for the 1-D case. However, it is not necessary to use planar holograms. Higher propagation angles that are needed to compensate the rotation of the eye can be obtained by using curved holograms or tiled and tilted SLMs or oblique incident light sources. Those configurations will be investigated in Section III-E. The increase in size and bandwidth associated with the desire to support a moving and rotating observer will also improve the viewing conditions of a stationary observer by providing the lower resolution peripheral view, and in turn, this will provide a more realistic experience.

2-D Hologram 3-D Image Reconstruction Case: The previous analysis was for the 1-D hologram case. Extension to 2-D hologram (3-D reconstruction) is rather straightforward. Assuming a circularly symmetric resolution capability for the human eye, we conclude that a circular hologram of a shape $A_{\min }$ is sufficient for a stationary observer [Fig. 8(a)]. Moreover, for an observer with a 2-D transversal motion in a parallel plane within area of shape $A_{O}$ [Fig. 8(b)], the minimum hologram size and the shape are obtained by morphologically dilating $A_{\min }$ by $A_{0}$ : $A_{H}=A_{\min } \oplus A_{O}$ as shown in Fig. 8(c). Morphological dilation operation can be found in [61]. Adding 2-D rotations will increase both the spatial extent (area) and the maximum frequency along those directions, similar to the 1-D case. Multiplication of the area by the obtained 2-D bandwidth will yield the SBP. In case of separable functions, extension to 2-D hologram from 1-D is even easier.

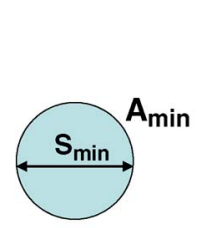

(a)

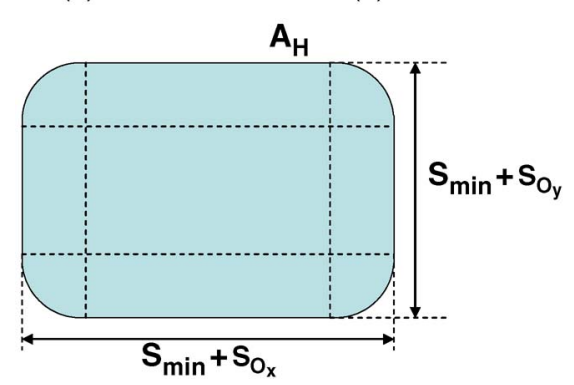

(c)

Fig. 8. (a) Minimum hologram size for a stationary observer. (b) Example for the allowed transversed motion range $A_{O}=S_{O X} \times S_{O y}$. (c) The shape of the minimum hologram is found as $A_{H}=A_{\min } \oplus A_{O}$ where $\oplus$ denotes the morphological dilation.
2) Pixel Period: Usually, SLMs, i.e., microdisplay panels, consist of pixels. Assuming rectangularly shaped pixels that are arranged also over a rectangular lattice, we write the pixelated function $g_{p}(x, y)$ of the bandlimited original data $g(x, y)$ on an SLM with $2 \mathrm{~N} \times 2 \mathrm{M}$ pixels as

$$
\begin{aligned}
g_{p}(x, y)= & \left(\sum_{n=-N}^{N-1} \sum_{m=-M}^{M-1} g(x, y) \delta\left(x-n \Delta p_{x}, y-m \Delta p_{y}\right)\right) \\
& * *\left[\operatorname{rect}\left(\frac{x}{\Delta p_{a x}}\right) \operatorname{rect}\left(\frac{y}{\Delta p_{a y}}\right)\right] \\
= & \left(\sum_{n=-N}^{N-1} \sum_{m=-M}^{M-1} g\left(n \Delta p_{x}, m \Delta p_{y}\right)\right. \\
& \left.\times\left[\operatorname{rect}\left(\frac{x-n \Delta p_{x}}{\Delta p_{a x}}\right) \operatorname{rect}\left(\frac{y-m \Delta p_{y}}{\Delta p_{a y}}\right)\right]\right)
\end{aligned}
$$

where $\Delta p_{x}$ and $\Delta p_{y}$ are the pixel periods on the SLM at the $(x, y)$ plane, and $\Delta p_{a x}$ and $\Delta p_{a y}$ are the active pixel sizes along $x$ and $y$, respectively [62]. The symbol $* *$ represents the 2-D convolution, $\delta(\cdot, \cdot)$ is the 2-D impulse function, and $\operatorname{rect}(\cdot)$ is the rectangular function, which is defined as

$$
\operatorname{rect}(x)= \begin{cases}1, & \text { for }-1 / 2 \leq x<1 / 2 \\ 0, & \text { else. }\end{cases}
$$

To successfully recover the original image by simple low-pass filtering (Shannon case), it is necessary to take samples close enough to each other to enable separation of the overlapping replicas in the spectral regions of $G_{p}\left(k_{x}, k_{y}\right)$, which is the Fourier transform of $g_{p}(x, y)$; this assures no aliasing. To determine the maximum allowable separation between the samples, we assume that $k_{x \max }$ and $k_{y \max }$ are the maximum frequencies (radians/unit length) along the $x$ and $y$ directions, respectively, and a rectangular frequency band around the origin. Since the centers of the replicas in the spectrum $G_{p}\left(k_{x}, k_{y}\right)$ are separated by $2 \pi / \Delta p_{x}$ and $2 \pi / \Delta p_{y}$ along the $k_{x}$ and $k_{y}$ directions, respectively, the no-aliasing condition is satisfied if the pixel periods are chosen as

$$
\Delta p_{x} \leq \frac{2 \pi}{2 k_{x \max }} \quad \text { and } \quad \Delta p_{y} \leq \frac{2 \pi}{2 k_{y \max }} .
$$

If we assume that the pixelated function $g_{p}(x, y)$ (i.e., the SLM) has an infinite extent, the maximum pixel periods for the exact recovery of the original function by low-pass filtering are thus $\left(\pi / k_{x \max }\right)$ and $\left(\pi / k_{y \max }\right)$, which are the sampling intervals associated with the Nyquist frequencies. The effect of sampling to the angular distribution of 
propagating light away from the SLM and its effect on the observed pattern are shown in Fig. 9.

We would like to add that Shannon recovery (i.e., recovery by low-pass filtering) is just one possible recovery procedure. If we may use other recovery techniques than simple low-pass filtering used for the Shannon sampling case, the Nyquist rate restriction above may not be needed. Indeed, Onural reported that objects can still be fully recovered even from severely undersampled (sampling below the Nyquist rate) Fresnel diffraction patterns [63]. The condition for full recovery from undersampled diffraction patterns is not bandlimitedness, but other restrictions like space-limitedness of the object pattern. Thus, if the diffraction pattern of a finite-size object is sampled, we will observe replicas of the image of the object in the reconstruction (higher diffraction orders) [63], [64]. Then, if the sampling rate is increased, replicas will move away from each other and vice versa. Sampling rate can be decreased until those replicas just overlap. Then, by simply windowing in space we will get a fully recovered object function. As a result, we can conclude that space-limited (thus not bandlimited) objects, which are quite common in real-life applications, can still be fully recovered from their below Nyquist rate samples. A generalization is the finitesupport limitation at a Fresnel domain with a specific parameter, as described by Gori [65], and this is equivalent to finite-support limitation in a corresponding fractional Fourier domain.

Returning back to the Shannon recovery case, we start with the bandwidth limitation that stems from the human visual system as mentioned earlier. We can apply the foregoing discussion (Shannon recovery) to various practical cases to find out the required maximum pixel period values. For example, for a stationary observer looking directly

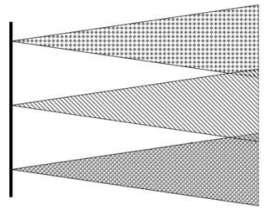

(a)

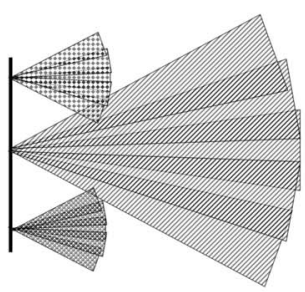

(c)

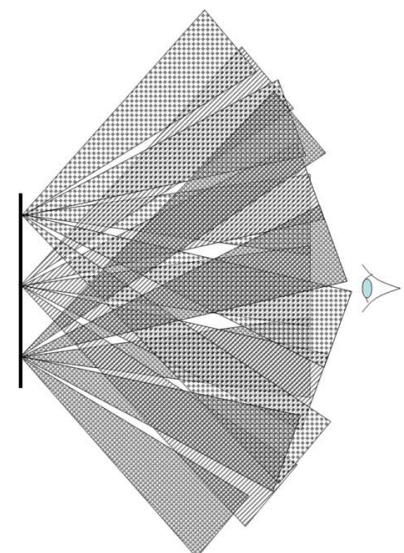

(b)

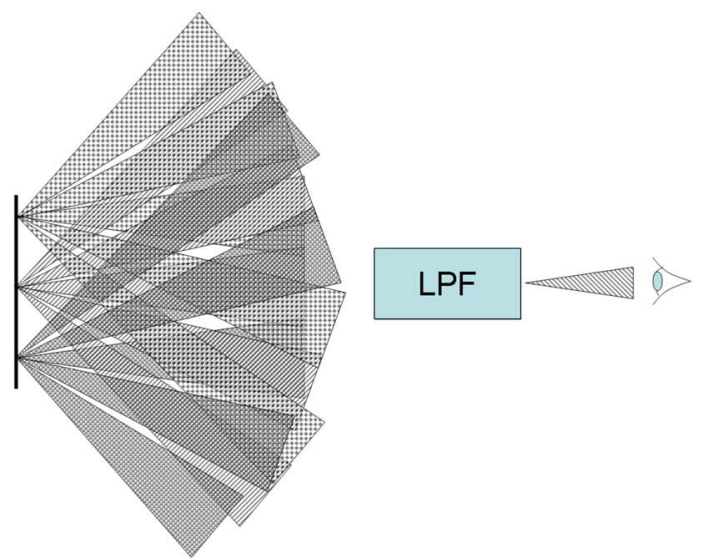

(d)

Fig. 9. Effect of pixel period on the angular distribution of diffracted light from a pixelated SLM and recovery by an optical low-pass filter. (a) Local bandwidth (propagation angle) associated with a hypothetical continuous (nonpixelated) SLM. Diffracted light propagates within a cone from each location on the SLM. (b) Consequence of the pixelated structure (sampling) to the propagation angle distribution (no aliasing case). Many well separated cones of light propagate from each SLM location. (c) Aliasing due to larger pixel size (coarse sampling). Propagating cones from each SLM location overlap. (Please note that cones propagate along the horizontal axis, indefinitely; only a portion is shown not to clutter the drawings. The extent of the cones has no relevance.) (d) Optical low-pass filter to recover the original image observed in (a), from (b). only low angle propagation passes through the filter at each SLM point (and low-pass filter) location. 
to the hologram (as in Fig. 6) from a distance of $1 \mathrm{~m}\left(D_{H}=\right.$ $1 \mathrm{~m}$ ), the maximum sampling interval is (for $\lambda=633 \mathrm{~nm}$ and $\left.\theta_{\max }=\phi_{v}=1.5^{\circ}\right) \Delta_{p}=\lambda /\left[2 \sin \left(\theta_{\max }\right)\right] \approx 12 \mu \mathrm{m}$ and $S_{\min }=5 \mathrm{~cm}$ from (1), (2), and (6).

However, if the observer is free to move and rotate, the bandwidth and the size of the hologram should be significantly increased. For example, for an observer $1 \mathrm{~m}$ away from the holographic display $\left(D_{H}=1 \mathrm{~m}\right)$ who can move $20 \mathrm{~cm}\left(S_{O}=20 \mathrm{~cm}\right)$ along the lateral direction and can rotate around the reconstruction in an angle of $\pm 15^{\circ}$ $\left(\theta_{R}=15^{\circ}\right)$, the required diffraction angle will be $\theta_{R}+$ $\theta_{\max }=16.5^{\circ}$ (where $\theta_{\max }=1.5^{\circ}$ ). Thus, the maximum pixel period will be $\Delta_{p}=\lambda /\left[2 \sin \left(\theta_{R}+\theta_{\max }\right)\right] \approx 1.1 \mu \mathrm{m}$ and the required display size will be $0.80 \mathrm{~m}\left(S_{H}=S_{O}+\right.$ $\left.2 \tan \left(\theta_{R}+\theta_{\max }\right) D_{H}\right)$. For a digital hologram whose size is $0.80 \mathrm{~m}$ and pixel period is $1.1 \mu \mathrm{m}$, the required number of pixels along one axis is more than 727000 .

The discussion above involves the sampling of the hologram due to the pixelated nature of the SLM and a reconstruction from it. Obviously, a procedure for recovering a continuous signal from the sampled signal is needed. Since wave propagation from one plane to another can be modeled as a linear shift invariant system, the order of wave propagation and low-pass filtering may be interchanged; thus a low-pass filtering at the observer end is sufficient. A complete recovery needs an optical low-pass filter whose physical location also moves along the transversal direction together with the observer. The structure for the optical low-pass filter is shown in Fig. 9(d). The bandwidth of the filter must be adjusted to pass the components whose frequencies are less than half of the pixel (sampling) frequency. Such an optical low-pass filtering will eliminate all high-order diffractions, as expected. However, the filter will also impose a narrow viewing angle (keyhole effect). In practice, the human eye naturally provides some low-pass filtering, but its bandwidth may be larger than the optical filter described above.

Instead of a low-pass filter during the reconstruction, an alternative way to avoid the unwanted effects of interfering higher frequency (angle) replications due to the pixelated structure of the SLMs is to increase the separation between the replicas, by smaller size pixels, so that no superposition of replicas is experienced by the observer. This is possible as a consequence of limited SLM and observation plane sizes. Therefore, the system parameters have to be chosen such that high-diffraction orders do not overlap within the observation plane. Fig. 10 shows the separation of the high-diffraction orders in the reconstructed field. The distance between the reconstruction plane and the hologram plane is $D_{R}$ and reconstruction size is $S_{\text {Rec }}$. The angle of the higher diffraction orders is $\theta_{O m}=\sin ^{-1}(m \lambda / \Lambda)$, where $\lambda$ is the wavelength of the light, $\Lambda$ is the period of the diffraction grating, and $m$ is the index of the diffraction order [66]. As seen in Fig. 10, to avoid overlapping of the high-diffraction orders, $\tan \left(\theta_{O 1}\right)$ should be larger than (approximately) $S_{\text {Rec }} / D_{R}$. We get

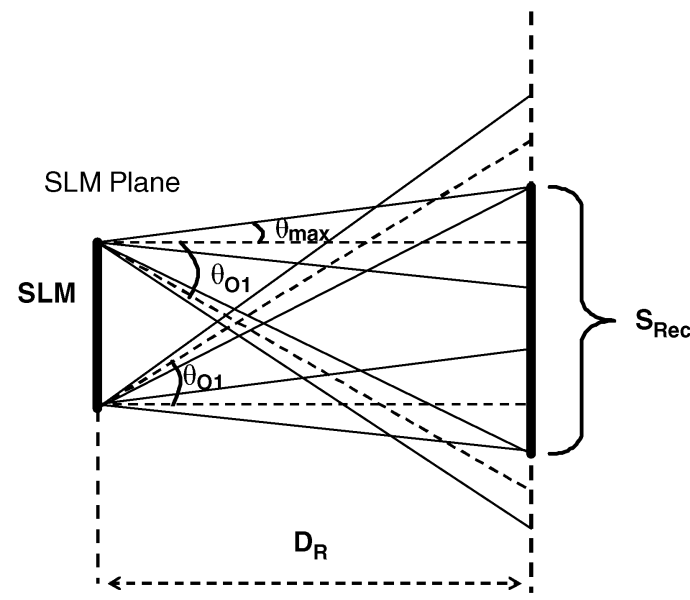

Reconstruction field

Fig. 10. High-diffraction orders in reconstruction field and their separation.

$S_{\text {Rec }}$ from the previous discussions, as, $S_{\text {Rec }}=S_{H}+$ $2 \tan \left(\theta_{\max }\right) D_{R}$.

For example, if the distance between the observer and the hologram is $1 \mathrm{~m}\left(D_{H}=1 \mathrm{~m}\right)$, and the observer is free to move along the lateral direction by $15 \mathrm{~cm}\left(S_{O}=15 \mathrm{~cm}\right)$, the required hologram size will be $20.2 \mathrm{~cm}\left(S_{H}=20.2 \mathrm{~cm}\right.$ and $\left.\theta_{\max }=1.5^{\circ}\right)$. If the distance between the SLM and the holographic reconstruction is $0.5 \mathrm{~m}\left(D_{R}=0.5 \mathrm{~m}\right)$, the reconstruction size will be $22.8 \mathrm{~cm}\left(S_{\operatorname{Rec}}=22.8 \mathrm{~cm}\right)$. If the frequency content of the digital hologram is fixed, in order to avoid overlapping of the high-diffraction orders, $\theta_{O 1}$ should be larger than $\tan ^{-1}\left(S_{\mathrm{Rec}} / D_{R}\right) \approx 24.5^{\circ}$. Since $\theta_{01}=\sin ^{-1}(\lambda / 2 \Delta p)$, the pixel period $\Delta p$ should be smaller than $0.76 \mu \mathrm{m}$ (for $\lambda=633 \mathrm{~nm}$ ).

With the help of this example, we can conclude that even without eye rotation (i.e., $\theta_{R}=0$ ) pixel period may fall into the subwavelength range if we want to avoid overlappings due to higher diffraction orders. Such small pixel sizes may not be feasible because of other physical constraints.

The discussion in this section is essential to understand the needed pixel period and the hologram size associated with planar SLM geometries under different viewing conditions. We see that the pixel size and the number of pixels may be prohibitive except for the simple and unrealistic case of a stationary observer. We should note, however, that this is primarily due to needed larger diffraction angles, especially from the edges of a planar SLM configuration. This also hints that nonplanar configurations are superior to support rotational motion since there will be no need for high-density pixels in that case.

Analysis in this section indicates that the resultant large SBP, which is prohibitive for current SLM and pixel sizes, may be relieved by implementing an adaptive systems that tracks the observer location and adjusts the 

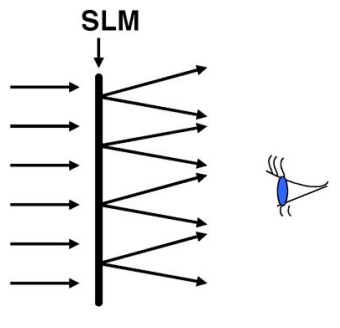

(a)

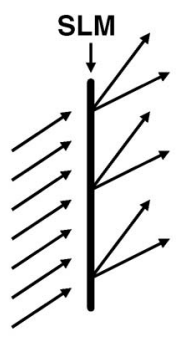

(b)
Fig. 11. (a) Orthographic illumination. (b) Tilted illumination.

illumination angle of the SLM, instead of a stationary orthographic illumination (see Fig. 11). Thus, the smaller bandwidth is shifted around a center frequency as indicated by the illumination angle. Therefore, instead of an $\mathrm{SBP}$ of $B W \times S_{\max }$, an SBP of $B W \times S_{H}$ at a time may still be sufficient. The commercial prototype described in [23][27] is based on such an approach.

\section{B. SBP as a Quality Metric for Holographic Reconstructions}

We have already discussed the needed SBP on the SLM surface to have satisfactory quality reconstructions. Information supported by the SLM as a holographic pattern on it is dispersed to the free space away from the SLM by the modulated light. Now we want to understand how that information is concentrated (or distributed) in space as a consequence of the diffraction during the reconstruction. First, the reconstruction space corresponding to a single planar SLM will be investigated.

As a plane wave is incident on a planar SLM perpendicularly, the light is diffracted and propagated to form the reconstruction corresponding to the input image (pattern on the SLM) within a limited angle due to the bandwidth of the system. The volume covered by the diffracted beam may be regarded as the reconstruction space. Fig. 12 shows the quality metric of the holographic reconstruction as a function of reconstruction position with respect to the SLM, for a given SLM size. (Note that this figure does not show a diffraction pattern; instead, it is just a pictorial representation of the variation of the profile of the quality

$850 \mathrm{~mm}$

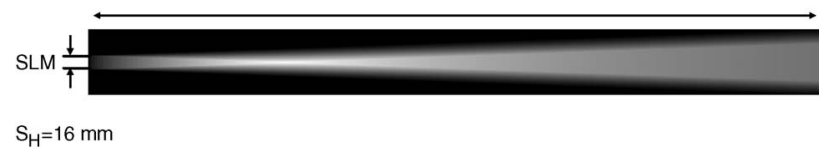

Fig. 12. Quality metric of reconstruction by the hologram on the SLM as a function of distance of the reconstructed image (white: high quality; black: low quality). metric as a function of the distance from the SLM.) Quality metric in this analysis basically shows how the ability of the SLM to concentrate information at a particular reconstruction position varies. By the help of this quality metric, we can find the optimum location that has the potential to yield maximum information concentration and therefore gives the highest quality local reconstructions that the system can provide. Frequency band of the system is equivalent to the solid angle in which the diffracted light propagates. Considering the distance of a focused point on the optical axis by the hologram on the SLM as a variable, we see that the bandwidth is restricted by the capabilities of the SLM until a certain distance, which we call $z_{0}$, is reached. The range $0<z<z_{0}$ corresponds to the case where the bandlimited quadratic phase pattern (the bandlimited Fresnel hologram of a point) remains entirely in the SLM [see Fig. 13(a) and (b)]. Therefore, for $0<z<z_{0}$, the bandwidth stays constant; however the area on the SLM that is covered by the bandlimited quadratic phase function increases with $z^{2}$ in this range. Therefore, the quality metric, which is the SBP of the reconstruction, gets better proportional to $z^{2}$ in $0<z<z_{0}$.

However, if $z$ is increased beyond $z_{0}$, portion of the related quadratic phase pattern on the hologram plane will not fall onto the SLM [see Fig. 13(c)], and thus the supported band will start decreasing. Assuming that the SLM sits entirely within the enlarged quadratic phase pattern for $z>z_{1}$, it is easy to see that the 2-D frequency band will start decreasing with $z^{2}$. The range $z_{0}<z<z_{1}$ will be the transition range.

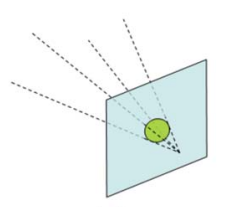

(a)

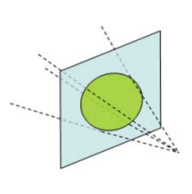

(b)
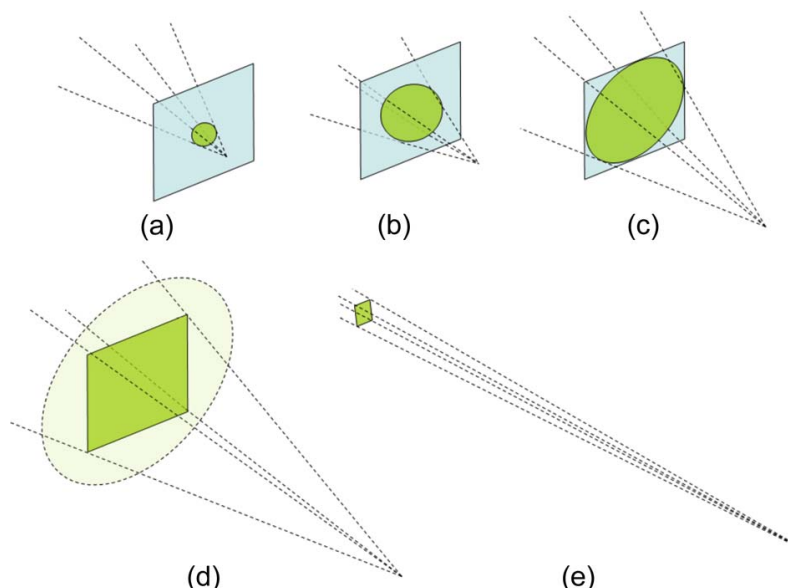

(d)

Fig. 13. Variation of "space" and "bandwidth" with respect to reconstruction distance $z$. (a) When $z$ is small, "space" is also small and the "band" is limited by the maximum band supported by the SLM. (b) As z moderately increases, "space" also increases and "band" is still limited by the maximum band supported by the SLM. (c) Transition. (d) For larger $z$, "space" does not change anymore, but "band" decreases since the supported diffraction angle (and therefore the spatial frequency) decreases. (e) For very large $z$, "space" does not change, but "band" decreases even further. 


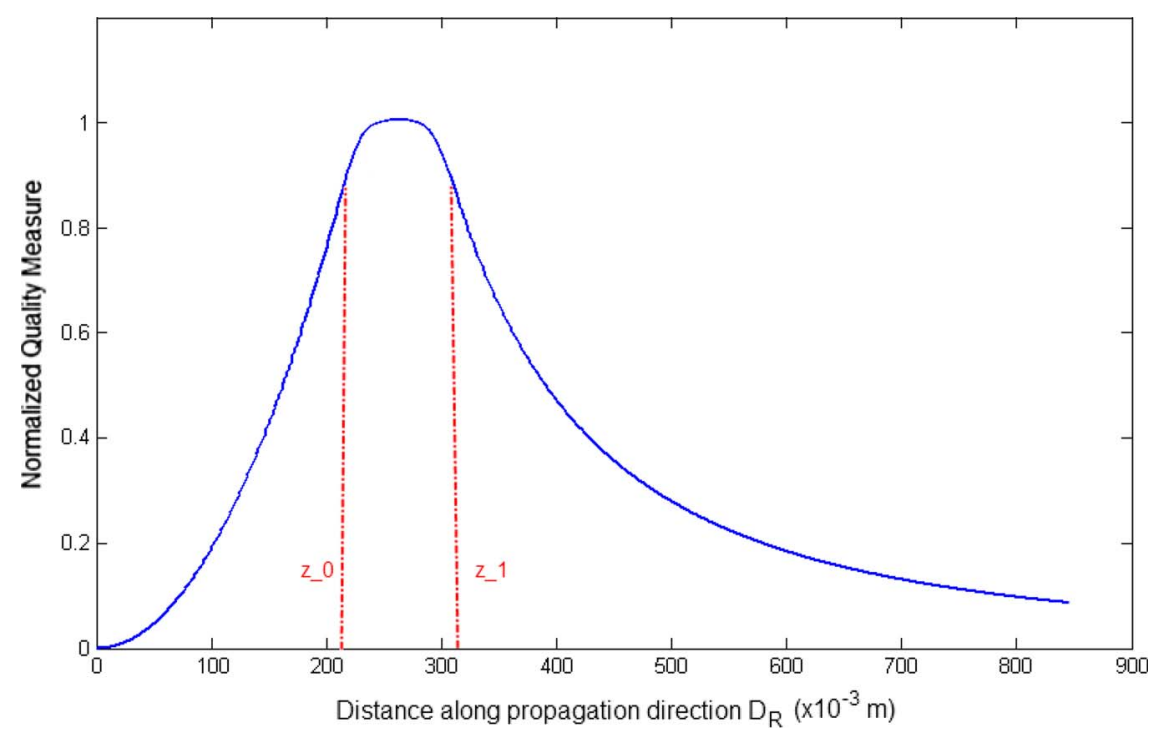

Fig. 14. Variation of normalized quality metric for a reconstructed image from a finite size SLM along the optical axis.

The variation of the quality metric along the optical axis is shown in Fig. 14. For an SLM whose dimension is $16 \mathrm{~mm}$ and pixel period is $9 \mu \mathrm{m}$ (i.e., $\theta_{\max }=2^{\circ}$ ) and for the wavelength $\lambda=633 \mathrm{~nm}$, the optimum reconstruction distance from hologram plane $D_{R}$ according to the SBP is approximately $228 \mathrm{~mm}$ (Fig. 14). In other words, for a given SLM size and pixel period, quality metric gives the reconstruction distance for best local holographic reconstruction.

We will extend this approach to a circularly configured multiple SLM holographic display in Section III-E.

\section{Noise and Quantization}

Theoretically, diffraction distributes the information due to a compact object to the entire space. As a consequence of that, reconstructions from even harshly quantized holograms may give reasonable results. Although holograms are quite immune to noise and quantization, it is still good to know the noise sources in digital holography. In commonly used pixelated electro-optical devices, fringe patterns are usually quantized to 256 levels. For example, in available phase-only SLMs, it is typical to have the phase quantized to 256 levels between 0 and $2 \pi$ radians. Mills and Yamaguchi reported some quantization effects in phase-shifting holography [67]. After numerical and experimental results they concluded that even 4-b quantization is enough, and, the difference when the quantization level is increased to 6-b or 8-b quantization is unnoticeable. Another source of error is the finite precision arithmetic used during the computation of holographic patterns. The physical setup itself that consists of optical elements is another source of distortion due to imperfections and imprecise alignments. Many optical elements have color aberration problem, which means they act differently for each wavelength. For example, focal length of the lenses may differ for different wavelengths. Multiple surface elements such as beam splitters and prisms also create some distortion on the reconstructions.

Another type of noise in digital holography is the speckle noise. When coherent light passes through or reflects back from randomly diffused media, randomized phase regions are generated and the superposition of such random phase components creates the speckle noise [68]. There are many methods to eliminate this undesirable effect [69]-[73]. LEDs have both time and space coherence to some extent. Narrow spectrum of LEDs brings some time coherence and using a pinhole in front of them increases the spatial coherence. However, since LEDs do not generate a perfectly coherent light, there is no observable speckle noise. LEDs are not harmful to the eyes as lasers if they are not too bright [74]. Therefore, reconstructed images can be observed by the naked eye under the LED illumination. Other advantages of LEDs are ease of operation and their low cost. However, due to low coherence characteristics reconstructions might be somewhat blurred.

Based on the discussions above we can conclude that higher than 4-b quantization gives satisfactory results for a human observer especially when the number of hologram pixels is high. Since commercially available SLMs generally use 8-b quantization, it is more than needed. Moreover, since LEDs are not fully coherent, decrease in the speckle noise is significant and holographic reconstructions by using LEDs are promising [49]-[52]. Therefore, LEDs can be effectively used in holographic display systems.

\section{Off-Axis Case}

Device specifications when using off-axis holograms should also be investigated. Off-axis holograms are used to shift the reconstructed image away from the undiffracted 


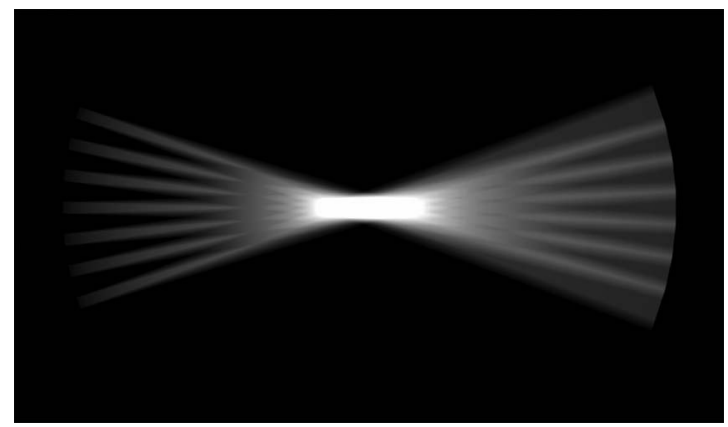

Fig. 15. Space variation of the quality metric for piecewise planar circular SLM configuration with multiple planar SLMs. The bright region at the center is the highest quality reconstruction zone. SLMs are at the left end of bright bands. Observer is at the right-hand side (white: high quality; black: low quality).

beam; otherwise, the observer would see the image in front of a bright background light source. Low-power undiffracted beam is annoying; a high-power undiffracted beam totally prevents visibility. In off-axis holography, the reference beam angle is chosen according to desired shift between the undiffracted beam and the desired reconstruction. However, this also shifts the frequency band of the holographic pattern since the technique is equivalent to a modulation of a signal by a carrier whose frequency is related to the angle of the reference beam. If the reference beam angle (off-axis) in the vertical direction is denoted as $\theta_{T}$, the maximum spatial frequency will increase by an additional $\sin \left(\theta_{T}\right) / \lambda$. For example, for the stationary observer looking directly to the hologram (as in the example in Section III-A2), if we use an off-axis hologram with a tilt angle of $2^{\circ}$ in vertical direction, we get $f_{\max }=\sin \left(1.5^{\circ}\right) /$ $\lambda+\sin \left(2^{\circ}\right) / \lambda=96$ cycles $/ \mathrm{mm}$. Then, the pixel period becomes $\Delta_{p} \approx 5 \mu \mathrm{m}$. Therefore, we can conclude that using off-axis hologram may change the required pixel size and spacing specifications significantly.

\section{E. Cylindrically Arranged Electroholographic Display System}

Electroholographic display systems are currently mostly planar, but they can be configured in different geometries as well. Planar configurations based on SLMs are quite common in the literature [24], [37], [75], [76]. Since planar holographic displays require more SBP to support rotations, necessity of using circular configuration is obvious. In a practical electroholography setup, a curved configuration consisting of properly aligned SLMs, each with a planar geometry, is a solution [28]. A wide angle reconstruction using such a system is also demonstrated at
Bilkent University (see Fig. 3). Thus, high-propagation angles can be achieved by tilting the SLM itself instead of increasing the pixel density. As a consequence of such a design the required pixel size can be kept quite coarse. The diffracted fields from each SLM overlap at the center of such a circularly configured SLM layer. A reconstruction at the optimal location will yield an excellent 3-D image, which can be observed from a large angle of view (Fig. 15). Moreover, the bandwidth of the system increases with the number of tilted SLMs since the tilted SLMs provide higher propagation angles to the observer. However, if the gap between the SLMs (generally formed by the mount of the SLM) is large, there might be discontinuities while changing the view from one SLM to another.

\section{CONCLUSION}

Digital holographic video displays are strong candidates for rendering ghost-like "true 3-D" motion images. Interest in this technology is increasing among the research community. Many laboratories have already reported different designs with promising results. Most of these designs are based on SLMs; SLMs with different capabilities and specifications have been used. Therefore, it is important to understand the limitations of such devices, and their effects on the resultant 3-D images. The two major parameters are the size and the resolution; therefore, the SBP is a suitable metric to assess a digital holographic device.

Reasonable sizes and resolutions seem to be sufficient for a stationary observer with no lateral or rotational motion. However, the needed SLM size and pixel density quickly increase beyond the capabilities of today's electronic technology when such motion is allowed as in a natural viewing environment. An alternative is to arrange planar SLMs on a curved mount to relieve the requirement of small and high-density pixels.

Since the holograms are quite robust to quantization errors, and since frame refresh rates are satisfactory for continuous perception, the focus of research is rather on designing digital holographic display sets, which can effectively support more freedom in lateral and rotational motion of the observer while providing satisfactory quality 3-D images.

\section{Acknowledgment}

The authors thank Dr. C. Falldorf for fruitful discussions that led to a better understanding and thus improvement of the analysis based on the human visual system and the image formation on the retina while looking at an image reconstructed by a hologram.

\section{REFERENCES}

[1] L. Onural, "Television in 3-D: What are the prospects?" Proc. IEEE, vol. 95, no. 6, pp. 1143-1145, Jun. 2007.
[2] M. Levoy, "Light fields and computational imaging," Computer, pp. 46-55, Aug. 2006.

[3] E. Sahin and L. Onural, "A comparative study of light field representation and integral imaging," Imag. Sci. J., vol. 58, pp. 28-31, 2010.

[4] P. Hariharan, Optical Holography: Principles, Techniques and Applications, 2nd ed. 
Cambridge, U.K.: Cambridge Univ. Press, 1996.

[5] D. Gabor, "A new microscopic principle," Nature, vol. 161, pp. 777-778, 1948.

[6] D. Gabor, "Microscopy by reconstructed wavefronts," Proc. R. Soc. A, vol. 197, pp. $454-487,1949$.

[7] D. Gabor, "Microscopy by reconstructed wavefronts: II," Proc. R. Soc. B, vol. 64 , pp. 449-469, 1951.

[8] E. N. Leith and J. Upatnieks, "Reconstructed wavefronts and communication theory," $J$. Opt. Soc. Amer., vol. 52, pp. 1123-1130, 1962

[9] E. N. Leith and J. Upatnieks, "Wavefront reconstruction with continuous-tone objects," J. Opt. Soc. Amer., vol. 53, pp. 1377-1381, 1963.

[10] E. N. Leith and J. Upatnieks, "Wavefront reconstruction with diffused illumination and three-dimensional objects," J. Opt. Soc. Amer., vol. 54, pp. 1295-1301, 1964.

[11] Y. N. Denisyuk, "Photographic reconstruction of the optical properties of an object in its own scattered field," Sov. Phys. Dokl., vol. 7, p. 543, 1962.

[12] T. Kreis, Handbook of Holographic Interferometry: Optical and Digital Methods. New York: Wiley, 2005.

[13] A. W. Lohmann and D. Paris, "Binary Fraunhofer holograms generated by computer," Appl. Opt., vol. 6, pp. 1739-1748, 1967.

[14] J. W. Goodman and R. W. Lawrence, "Digital image formation from electronically detected holograms," Appl. Phys. Lett., vol. 11, pp. 77-79, 1967.

[15] L. P. Yaroslavskii and N. S. Merzlyakov, Methods of Digital Holography. New York: Consultants Bureau, 1980.

[16] F. Yaras, M. Kovachev, R. Ilieva, M. Agour, and L. Onural, "Holographic reconstruction using phase-only spatial light modulators," in Proc. 3DTV Conf., The True Vision - Capture, Transmission and Display of 3D Video, 2008, DOI: 10.1109/3DTV.2008.4569826.

[17] K. Bauchert, S. Serati, and A. Furman, "Advances in liquid crystal spatial ligh modulators," Proc. SPIE - Opt. Pattern Recognit. XIII, vol. 4734, pp. 35-43, 2002.

[18] A. Michalkiewicz, M. Kujawinskaa, T. Kozackia, X. Wangb, and P. J. Bosb, "Holographic three-dimensional displays with liquid crystal on silicon spatial light modulator," Proc. SPIE-Interferometry XII: Tech. Anal., vol. 5531, pp. 85-94, 2004.

[19] N. Ohmura, H. Kang, T. Yamaguchi, and H. Yoshikawa, "A method to increase the hologram viewing angle by the beam reconfiguration," Proc. SPIE-Practical Holography XXII: Mater. Appl., vol. 6912, 691200, 2008.

[20] R. Tudela, I. Labastida, E. Marti-Badosa, S. Vallmitjana, I. Juvells, and A. Carnicer, "A simple method for displaying fresnel holograms on liquid crystal panels," Opt. Commun., vol. 214, pp. 107-114, 2002.

[21] R. Tudela, E. Martin-Badosa, I. Labastida, S. Vallmitjana, I. Juvells, and A. Carnicer, "Full complex fresnel holograms displayed on liquid crystal devices," J. Opt. A, Pure Appl. Opt., vol. 5, pp. 189-194, 2003.

[22] R. Tudela, E. Martin-Badosa, I. Labastida, S. Vallmitjana, and A. Carnicer, "Wavefront reconstruction by adding modulation capabilities of two liquid crystal devices," Opt. Eng., vol. 43, no. 11, pp. 2650-2657, Nov. 2004.

[23] A. Schwerdtner, R. Haussler, and N. Leister, "Large holographic displays for real-time applications," Proc. SPIE, vol. 6912, no. 1, 69120T, 2008.

[24] S. Reichelt, R. Haussler, N. Leister, G. Futterer, and A. Schwerdtner, "Large holographic 3D displays for tomorrow's TV and monitors-solutions, challenges, and prospects," in Proc. IEEE Lasers Electro-Opt. Soc. Annu. Meeting, 2008, pp. 194-195.

[25] R. Häussler, A. Schwerdtner, and N. Leister, "Large holographic displays as an alternative to stereoscopic displays," Proc. SPIE, vol. 6803, no. 1, 68030M, 2008.

[26] S. Reichelt, H. Sahm, N. Leister, and A. Schwerdtner, "Capabilities of diffractive optical elements for real-time holographic displays," Proc. SPIE, vol. 6912, no. 1, 69120P, 2008.

[27] N. Leister, A. Schwerdtner, G. Fütterer S. Buschbeck, J.-C. Olaya, and S. Flon, "Full-color interactive holographic projection system for large 3D scene reconstruction," Proc. SPIE, vol. 6911, no. 1, 69110V, 2008.

[28] J. Hahn, H. Kim, Y. Lim, G. Park, and B. Lee, "Wide viewing angle dynamic holographic stereogram with a curved array of spatial light modulators," Opt. Exp., vol. 16, no. 16, pp. 12372-12386, 2008.

[29] P. S. Hilaire, S. A. Benton, M. Lucente, and P. M. Hubel, "Color images with the MIT holographic video display," Proc. SPIE - Practical Holography VI, vol. 1667, pp. 73-84, 1992.

[30] P. St.-Hilaire, "Scalable optical architectures for electronic holography," Ph.D. dissertation, Program Media Arts Sci. Massachusetts Inst. Technol., Cambridge, CA, 1994.

[31] P. St-Hilaire, S. A. Benton, M. Lucente, J. D. Sutter, and W. J. Plesniak, "Advances in holographic video," Proc. SPIE-Practical Holography VII, vol. 1914, pp. 188-196, 1993.

[32] P. St.-Hillaire, M. Lucente, J. Sutter, R. Pappu, C. J. Sparrell, and S. Benton, "Scaling up the MIT holographic video system," in Proc. 5th Int. Symp. Display Holography, 1995, pp. $374-380$

[33] M. L. Jepsen, "Holographic video: Design and implementation of a display system," M.S. thesis, Dept. Architecture, Massachusetts Inst. Technol., Cambridge, MA, 1989.

[34] J. S. Kollin, "Design and information considerations for holographic television," M.S. thesis, Dept. Architecture, Massachusetts Inst. Technol., Cambridge, MA, 1988.

[35] M. Lucente and T. Galyean, "Rendering interactive holographic images," in Proc. 22nd Annu. Conf. Comput. Graph. Interactive Tech. 1995, DOI: $10.1145 / 218380.218490$.

[36] J. Underkoffler, "Towards accurate computation of optically reconstructed holograms," M.S. thesis, Program Media Arts Sci., Massachusetts Inst. Technol., Cambridge, MA, 1991.

[37] M. Stanley, M. A. Smith, A. P. Smith, P. J. Watson, S. D. Coomber, C. D. Cameron, C. W. Slinger, and A. D. Wood, "3D electronic holography display system using a 100 mega-pixel spatial light modulator," Proc. SPIE, vol. 5249, pp. 297-308, 2004.

[38] T. Shimobaba, S. Hishinuma, and T. Ito, "Special-purpose computer for holography HORN-4 with recurrence algorithm," Comput. Phys. Commun., vol. 148, no. 2 , pp. 160-170, 2002.

[39] T. Yabe, T. Ito, and M. Okazaki, "Holography machine HORN-1 for computer-aided retrieval of virtual three-dimensional image,"
Jpn. J. Appl. Phys. 2, Lett., vol. 32, no. 9, pp. L1359-L1361, 1993.

[40] Y. Ichihashi, T. Ito, H. Nakayama, N. Masuda, A. Shiraki, and T. Shimobaba, "Development of special-purpose computer HORN-6 for holography," in Proc. 3D Image Conf., 2008.

[41] T. Ito, N. Masuda, K. Yoshimura, A. Shiraki, T. Shimobaba, and T. Sugie, "Special-purpose computer HORN-5 for a real-time electroholography," Opt. Exp., vol. 13, no. 6, pp. 1923-1932, 2005.

[42] T. Shimobaba, N. Masuda, T. Sugie, S. Hosono, S. Tsukui, and T. Ito, "Special-purpose computer for holography HORN-3 with PLD technology," Comput Phys. Commun., vol. 130 , no. $1-2$, pp. $75-82$, Jul. 2000.

[43] T. Ito, T. Yabe, M. Okazaki, and M. Yanagi, "Special-purpose computer HORN-1 for reconstruction of virtual image in three dimensions," Comput. Phys. Commun., vol. 82, pp. 104-110, 1994.

[44] T. Ito, H. Eldeib, K. Yoshida, S. Takahashi, T. Yabe, and T. Kunugi, "Special-purpose computer for holography HORN-2," Comput. Phys. Commun., vol. 93, pp. 13-20, 1996.

[45] M. Schubin, "NAB 2009: Holography update Television Broadcast, Apr. 20, 2009. [Online]. Available: http://www.televisionbroadcast. com/article/79134

[46] F. Yaraş, H. Kang, and L. Onural, "State of the art in holographic displays: A survey," J. Display Technol., vol. 6, no. 10, pp. 443-454, 2010.

[47] L. Onural, G. Bozdağı, and A. Atalar, "New high-resolution display device for holographic three-dimensional video: Principles and simulations," Opt. Eng., vol. 33, pp. 835-844, 1994.

[48] R. W. Gerchberg and W. O. Saxton, "A practical algorithm for the determination of the phase from image and diffraction plane pictures," Optik, vol. 35, pp. 706-713, 1972.

[49] F. Yaraș and L. Onural, "Color holographic reconstruction using multiple SLMs and LED illumination,” Proc. SPIE, vol. 7237 723700, 2009.

[50] F. Yaras, H. Kang, and L. Onural, "Real-time phase-only color holographic video display system using LED illumination," Appl. Opt., vol. 48, no. 34, pp. H48-H53, 2009.

[51] F. Yaraș, H. Kang, and L. Onural, "Real-time multiple SLM color holographic display using multiple GPU acceleration," presented at the OSA Digital Holography Three-Dimensional Imaging, Vancouver, Canada, DWA4, 2009.

[52] F. Yaraș, H. Kang, and L. Onural, "Real-time color holographic video display system," in Proc. 3DTV Conf.: The True Vision-Capture Transmission Display 3D Video, 2009 DOI: 10.1109/3DTV.2009.5069660.

[53] H. Kang, "Quality improvements of the coherent holographic stereogram for natural 3D display and its applications," Dept. Electron. Eng., Ph.D. dissertation, Nihon Univ., Tokyo, Japan, 2008.

[54] H. Kang, F. Yaras, L. Onural, and H. Yoshikawa, "Real-time fringe pattern generation with high quality," presented at the OSA Digital Holography Three-Dimensional Imaging, Vancouver, Canada, DTuB7, 2009.

[55] H. Kang, F. Yaraş, and L. Onural, "Quality comparison and acceleration for digital hologram generation method based on segmentation," in Proc. 3DTV Conf.: The True Vision-Capture Transmission Display 3D Video, 2009, DOI: 10.1109/3DTV.2009. 5069661. 
[56] H. Kang, F. Yaraş, and L. Onural, "Graphics processing unit accelerated computation of digital holograms," Appl. Opt., vol. 48, no. 34 pp. H137-H143, 2009.

[57] R. L. D. Valois and K. K. D. Valois, Spatial Vision. Oxford, U.K.: Oxford Science Publications, 1990.

[58] M. F. Deering, "The limits of human vision," in Proc. 2nd Int. Immersive Projection Technol. Workshop, 1998.

[59] D. A. Atchison and G. Smith, Optics of the Human Eye. New York: Elsevier Health Sciences, 2000.

[60] L. Onural, A. Gotchev, H. M. Ozaktas, and E. Stoykova, "A survey of signal processing problems and tools in holographic three-dimensional television," IEEE Trans. Circuits Syst. Video Technol., vol. 17, no. 11, pp. 1631-1646, Nov. 2007.

[61] R. Gonzalez and R. Woods, Digital Image Processing. Reading, MA: Addison-Wesley, 1992, pp. 518-519.

[62] T. M. Kreis, "Frequency analysis of digital holography," Opt. Eng., vol. 41, no. 4, pp. 771-778, Apr. 2002.
[63] L. Onural, "Sampling of the diffraction field," Appl. Opt., vol. 39, pp. 5929-5935, 2000.

[64] L. Onural, "Exact analysis of the effects of sampling of the scalar diffraction field," J. Opt. Soc. Amer. A, no. 2, pp. 359-367.

[65] F. Gori, "Fresnel transform and sampling theorem," Opt. Commun., vol. 39, pp. 293-297, 1981.

[66] B. Saleh and M. Teich, Fundamentals of Photonics. New York: Wiley, 1991.

[67] G. A. Mills and I. Yamaguchi, "Effects of quantization in phase-shifting digital holography," Appl. Opt., vol. 44, no. 7 pp. 1216-1225, 2005.

[68] L. I. Goldfischer, "Autocorrelation function and power spectral density of laser-produced speckle patterns," J. Opt. Soc. Amer., vol. 55, no. 3, pp. 247-252, 1965.

[69] H. J. Gerritsen, W. J. Hannan, and E. G. Ramberg, "Elimination of speckle noise in holograms with redundancy," Appl. Opt., vol. 7, no. 11, pp. 2301-2311, 1968.

[70] J. M. E. Myers and A. M. Wims, "Elimination of speckle noise in laser light scattering photometry," Appl. Opt., vol. 11, no. 4, pp. 947-949, 1972
[71] M. Matsumura, "Speckle noise reduction by random phase shifters," Appl. Opt., vol. 14, no. 3, pp. 660-665, 1975.

[72] J. Amako, H. Miura, and T. Sonehara, "Speckle-noise reduction on kinoform reconstruction using a phase-only spatial light modulator," Appl. Opt., vol. 34, no. 17, pp. 3165-3171, 1995.

[73] J. M. Huntley and L. Benckert, "Speckle interferometry: Noise reduction by correlation fringe averaging," Appl. Opt., vol. 31, no. 14, pp. 2412-2414, 1992.

[74] Y. Barkana and M. Belkin, "Laser eye injuries," Surv. Ophthalmol., vol. 44, no. 6 , pp. 459-478, 2000.

[75] T. Yamaguchi, G. Okabe, and H. Yoshikawa, "Real-time image plane full-color and full-parallax holographic video display system," Opt. Eng., vol. 46, no. 12, 125801, 2007.

[76] H. Kang, N. Ohmura, T. Yamaguchi, H. Yoshikawa, S.-C. Kim, and E.-S. Kim, "Method to enlarge the hologram viewing window using a mirror module," Opt. Eng., vol. 48, no. 7, 075801, 2009.

\section{ABOUT THE AUTHORS}

Levent Onural (Fellow, IEEE) received the B.S. and M.S. degrees in electrical engineering from the Middle East Technical University (METU), Ankara, Turkey, in 1979 and 1981, respectively, and the Ph.D. degree in electrical and computer engineering from the State University of New York at Buffalo (SUNYAB), Buffalo, in 1985

He was a Fulbright scholar (1981-1985). He joined the Electrical and Electronics Engineering Department, Bilkent University, Ankara, Turkey, in

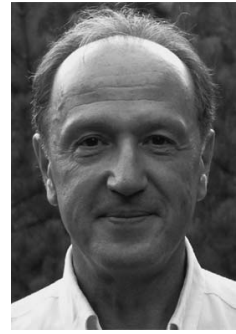
1987, where he is currently a Professor and Dean of Engineering. His current research interests are in image and video processing, with emphasis on video coding, 3DTV, holographic 3DTV, and signal processing aspects of optical wave propagation. He was the coordinator of European Commission (EC)-funded 3DTV Project (2004-2008), and the Coleader of 3D Media Cluster (2008-2010), which is an umbrella organization formed by many EC-funded 3-D related projects.

Dr. Onural is the recipient of a TüBiTAK Incentive Award (1995), an IEEE Third Millennium Medal (2000), and the 2011 IEEE Haraden Pratt Award. He served IEEE as Director of IEEE Region 8 (2001-2002), Secretary of IEEE (2003), and a member of IEEE Board of Directors (2001-2003).

Fahri Yaraș (Member, IEEE) received the B.S. degree in electrical and electronics engineering from Bilkent University, Ankara, Turkey, in 2005, where he is currently working towards the Ph.D. degree in electrical and electronics engineering.

He was a member of European Commission (EC)-funded 3DTV Project (2005-2008). Currently, he is a Researcher in EC-funded Real 3D Project. His research interests are 3DTV, holographic 3DTV, 3-D displays, electroholography. and optical signal processing.

Mr. Yaraș is a member of the Optical Society of America (OSA)
Hoonjong Kang received the B.Eng. and M.Eng. degrees in electrical engineering from Kwangwoon University, Seoul, Korea, in 1998 , and 2001, respectively, and the Ph.D. degree in electronics engineering from Nihon University, Tokyo, Japan, in 2008.

He worked in 3D Korea company (2000), and in ETRI (2002). He was a member of the Bilkent University, Ankara, Turkey, Real 3D project team (2008-2010). He joined the Korea Electronics Technology Institute (KETI), Gyeonggi, Korea, in 2010. His research interests include computer-generated holograms, electroholography, 3-D display, and signal processing of stereoscopic images. 